\title{
New Innovations in Testing Sustained-Release Tablets Using an Automated Dissolution System with Online Dilution
}

\author{
Huang Hai-wei ${ }^{1}$, Yuan Song ${ }^{1}$, Yu Li-ju${ }^{1}$, He Lan ${ }^{1}$, Zhang Qi-ming ${ }^{1}$, Ning Bao-ming ${ }^{1, *}$, Keith Wilkinson², \\ Wei Shi ${ }^{2}$, and Shi Li-fang ${ }^{2}$ \\ ${ }^{1}$ National Institutes for Food and Drug control, Beijing, China \\ ${ }^{2}$ Automated Lab Systems, Berkshire, UK
}

\section{ABSTRACT}

This paper describes the development and validation of an automated method for the dissolution testing of metformin hydrochloride for use in the quality assurance program. Automation could provide significant improvements in the consistency of results compared with existing manual methods. Care and consideration were given to the automation of preparation of samples and standards in line with current manual procedures. Reproducibility and sample preparation accuracy were quantified, and results obtained were compared against tests performed manually.

The system was validated in accordance with the Chinese (1), European (2), and United States pharmacopeias (3) using salicylic acid. Salicylic acid testing for bath calibration also required dilution, which provided a direct comparison to the automated system used to test metformin hydrochloride tablets.

KEYWORDS: Dissolution; automation; online; validation; method development.

\section{INTRODUCTION}

$\mathrm{M}$ etformin hydrochloride is an oral antidiabetic drug used in the treatment of type 2 diabetes. It is also used in the treatment of polycystic ovary syndrome and has been investigated for other diseases where insulin resistance may be an important factor. Metformin is the only antidiabetic drug that has been conclusively shown to prevent the cardiovascular complications of diabetes $(4,5)$. In China, metformin hydrochloride oral solid dosages are manufactured by nearly 200 companies. The current dissolution methodology for testing metformin hydrochloride oral solid dosage is to collect samples, replace the sampling volume removed with fresh medium, dilute the sample, and measure by UV spectrophotometry.

In China, the National Institute for Food and Drug Control (NIFDC) is responsible for the regulation and quality of drugs. This includes post-market surveillance sampling and specification evaluation of imported drugs as well as drugs that are produced in China. We now report on a novel method for dissolution testing of solid dose metformin hydrochloride using an automated technique that we believe has advantages over methodology currently used by the NIFDC.

\section{SYSTEM DESCRIPTION}

A schematic representation of the new method and system configuration is shown in Figure 1. It consists of the ADT8 dissolution bath from Automated Lab Systems (Berkshire, UK), an ALS 8 Syringe Pump (used for sampling), ALS 3-port Sample Loss Replacement and Sampling Valves,

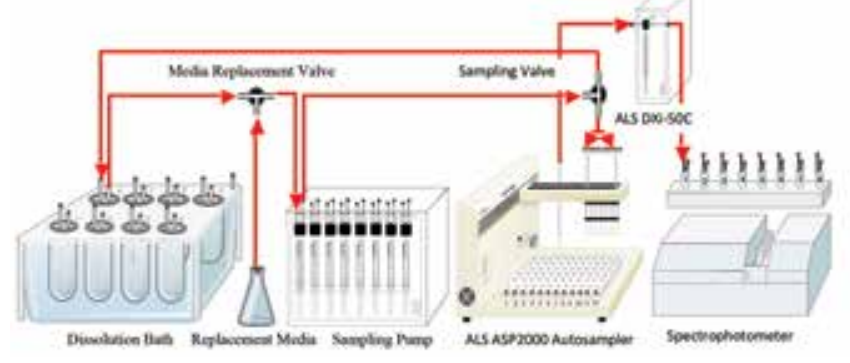

Figure1. Schematic of the ASP2000 automated system connected directly to the UV spectrophotometer.

ALS ASP2000 Dual Probe XYZ Robotic Sampler, ALS DXi50 Syringe Diluter, and PGI T70 Spectrophotometer (PG Instruments, Ltd.). Samples were filtered using in-line disc filters (Gelman Acrodisc GF $0.45-\mu \mathrm{m}$ ) configured outside the dissolution vessels with Luer fittings for easy removal and replacement. Metformin hydrochloride exhibits UV absorption linearity up to about $15 \mathrm{mg} / \mathrm{L}$ at about $233 \mathrm{~nm}$ (7); therefore, 500-mg tablets require approximately 100fold dilution, which is in line with current methodology (6).

The challenge for this test is diluting 1 in 100. The diluter is capable of single dilutions of 1 in 30 within $1 \%$ accuracy. Therefore, serial dilution was performed to achieve the higher dilution of 1 in 100. The ASP2000 XYZ Robotic Sampler fitted with two probes was used. Racks required for the analysis with serial dilution were configured using IDIS and ASP2000 teachable rack configuration, which allowed optimized positioning of samples to suit our requirements. Up to 44 time points, five stock standards, eight prepared standards, and two blanks, $100 \mathrm{~mL}$ each, can be configured for the ASP2000 Dual Probe XYZ Robotic Sampler. This 
virtual rack design allowed us to configure racks to realize the requirements to perform serial dilution. Three racks each accommodating 11 time points $(11 \times 7), 100-\mathrm{mL}$ blank, and eight standards were configured (Figure 2).

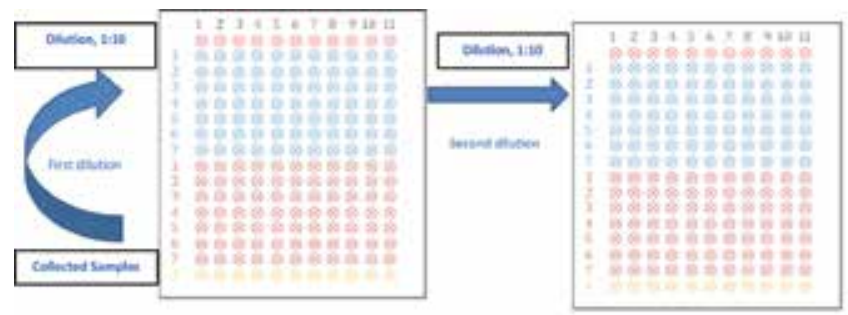

Figure 2. Schematic of racks configured to meet requirements for serial dilution.

The design of two probes realizes simultaneous collection and dilution using three-dimensional XYZ movement to access sample tubes anywhere on the ASP2000 Dual Probe XYZ Robotic Sampler. The left probe, with eight needles, was used to collect all samples simultaneously, while the right probe, with a single needle linked to the diluter, was used to dilute and inject the samples into the spectrophotometer.

The commercially available system is used by other companies for streamlining collection, dilution, and measurement for UV or HPLC automation. The schematic in Figure 3 shows the operation workflow. Prior to sampling, a selected volume of sample is pumped in circulation to the bath to equilibrate fresh sample solution in the lines. At the sampling time interval, the valve switches to the ASP2000 Multiple Probe, which moves to sampling tubes to dispense the desired volume of sample solution. The complete system is controlled by the IDIS software supplied by ALS. This software controls instruments from different manufacturers (baths from Agilent, Distek, Erweka, Pharmatest, Sotax; spectrophotometers from Agilent, Perkin Elmer, Jena, Shimadzu, Thermo; peristaltic and syringe pumps) and allows the user to configure methodologies using instruments from different sources for closed-loop UV analysis, collection, and HPLC analysis linked to ChemStation or Empower as well as to collect, prepare, and measure samples on UV followed by result calculations and reporting. Any combination of bath, pump, and spectrophotometer can be selected. Methodology is created by adding symbols representing the driver for the instruments onto the method desktop; linking the symbols by making connection to points on the symbols; and finally setting sampling times, temperature, and stirrer speed of the bath. During the analysis, raw data (absorbance in this case) acquired from the spectrophotometer, as well as temperature and stirrer speed from the bath, are stored to IDIS relational database immediately after collection.

The IDIS software optimizes the timings to make sure the time to the next sampling interval is not compromised. If there is enough time before the next samples are due, the ASP2000 single probe connected to the diluter is positioned at the appropriate tube and sample is aspirated, then the ASP2000 moves to another tube and the diluter dispenses the sample with diluent to perform dilution. If there is not enough time to perform dilution or injection and measurement, the system defers this sequence until after the next collection, a process referred to as Deferred Data Collection by ALS. In practice, this deferment can occur indefinitely, especially for short time intervals, in which case the processing (dilution, injection, and measurement) will be performed at the end of the dissolution analysis time (Figure 3).

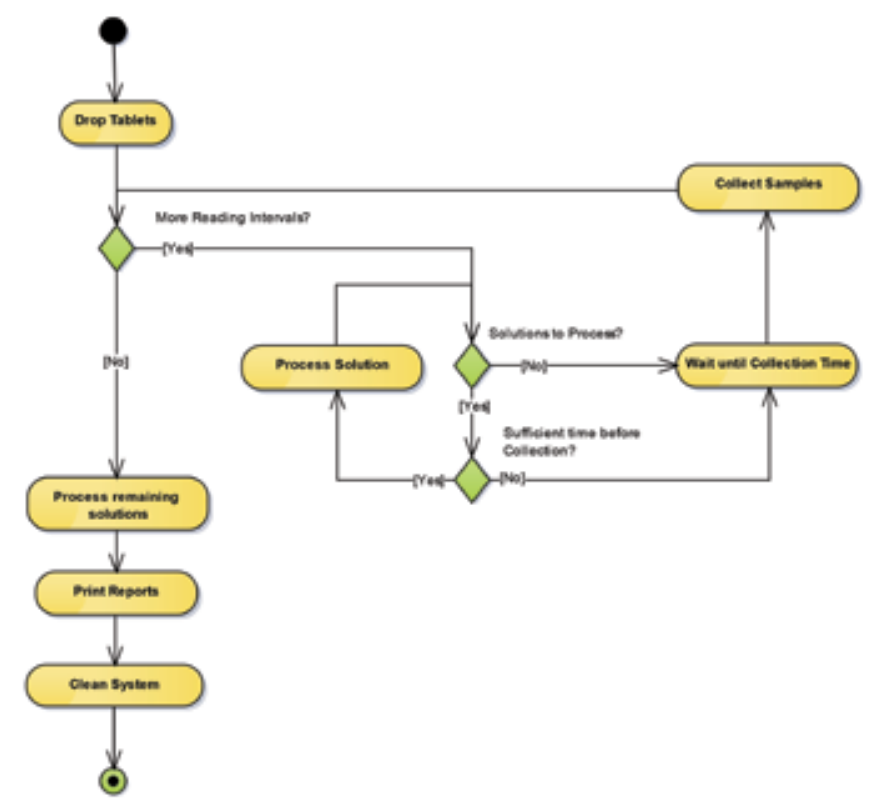

Figure 3. Flowchart of automated sequence.

Diluted solutions (sample, standard, and blanks) are aspirated then dispensed in the appropriate order into a port on the ASP2000 connected to a flow cell in the spectrophotometer, and measurements are performed on the spectrophotometer. Dissolution results are calculated and displayed in real time. The complete system is controlled by the IDIS software, which also manages the calculations and data record storage using an Oracle database. The entire sequence of operations (collection, filtration, media replacement, dilution, measurement, and calculation of results) is automated and requires no operator intervention.

\section{AUTOMATED DISSOLUTION TESTS FOR METFORMIN HYDROCHLORIDE}

Commercial samples of metformin hydrochloride extended-release 500-mg tablets were analyzed in accordance with USP (6) using the automated system described.

The test used $1000 \mathrm{~mL}$ of $\mathrm{pH} 7.4 \pm 0.05$ phosphate buffer $(6.8 \pm 0.1 \mathrm{~g}$ of potassium dihydrogen phosphate and $1.58 \pm 0.05 \mathrm{~g}$ of sodium hydroxide in $1000 \mathrm{~mL}$ of deionized degassed water). The system was configured with paddles $(1,2) ; 10 \mathrm{~mL}$ of sample was collected at each interval (six 
samples) at $15 \mathrm{~min}, 30 \mathrm{~min}$, and 1, 2, 3, 4, 6, 8, 10, 12, and $14 \mathrm{~h}$. After each sampling, the volume removed from the vessel was automatically replaced by the system.

The graphically configured method is shown by Figure 4. A method was configured to collect samples at multiple time points; prepare standards, blanks, and samples by dilution; and inject each solution into the UV. After injection, UV measurement was performed; dissolution results were calculated and displayed in real time. The complete process is streamlined and automated; this was not previously achievable.

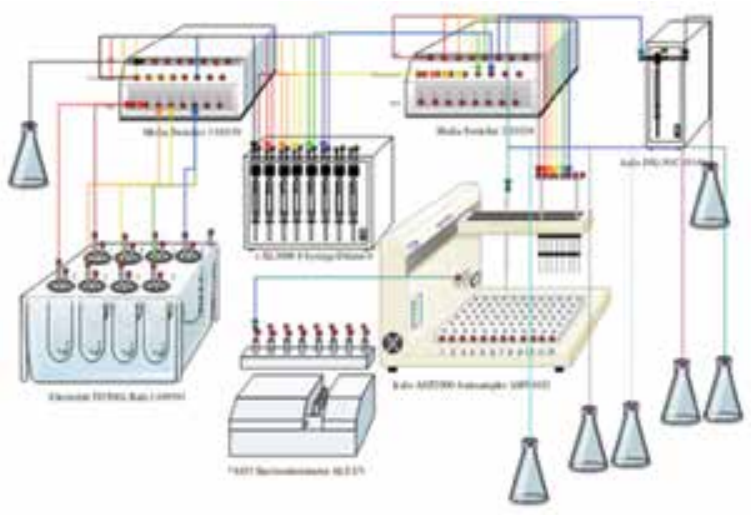

Figure 4. Method configuration schematic. Actual representation of the method configuration for the analysis.

Samples collected were diluted 1 in 10 , then further diluted 1 in 10 to achieve a dilution of 1 in 100, shown by schematic Figure 2 . Stock standards were prepared by accurately weighing and dissolving $10 \mathrm{mg}$ of metformin hydrochloride in $100 \mathrm{~mL}$ of DI water. Stock solutions were further diluted by the system (1 in 20), and the diluted standards and samples were injected and measured on the spectrophotometer at $233 \mathrm{~nm}$. Prior to reading standards and samples, blank measurements were performed. Dissolution results were calculated as the experiment proceeded, and the profile was displayed in real time.

Results and dissolution profiles were calculated and plotted in real time, and reports were generated automatically at the end of the analysis. The UV absorbance readings for Salicylic Acid are shown in Tables 1 and 2, and Table 3 shows the percent dissolved results for metformin hydrochloride.

\section{System Control and Synchronization of Collection with Sample Processing}

Full automation of dissolution has very complex procedures. The time points of sampling, dilution, and injection must be controlled precisely. If this is not coordinated, there will be timing conflicts between the processing performed in real time and collection, which must be precise according to pharmacopoeia.

The IDIS software controls the instruments and uses an innovative algorithm to coordinate and optimize scheduling to improve efficiency and avoid conflicts between the collection and the sample dilution and measurement times (Figure 3). Without the scheduler (deferred data collection), processes would have to be performed within large time intervals, which limits sampling at short time intervals.

\section{Compliance with Pharmacopeia Guidelines}

Strict quality control is required for automation of dissolution in NIFDC. The automated system complies with FDA 21 CFR guidelines, allowing use in pharmacopoeiacompliant laboratories, importantly quality control environments. The system provides four-level right access in the system: Administration, Development, Application, and Instrumentation with electronic signing.

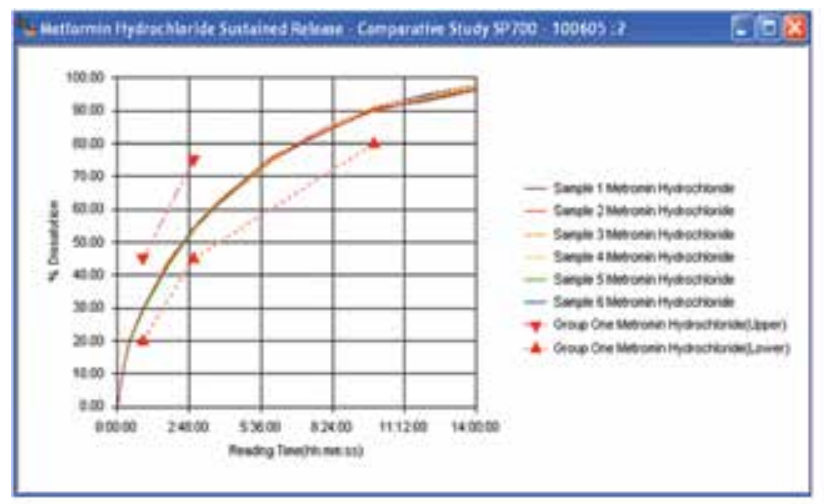

Figure 5. Percent dissolution graph from the automated system for metformin hydrochloride dissolution profile with high/low specification compliance error limits in red.

\section{VALIDATION}

The automated system was validated in accordance with the United States, European, and Chinese pharmacopoeias (1-3) using 300-mg salicylic acid tablets, LOT 100103-200610 (NIFDC, China), dissolved in $900 \mathrm{~mL}$ of $\mathrm{pH} 6.8$ phosphate buffer medium prepared with $6.8 \pm 0.1$ $\mathrm{g}$ of potassium dihydrogen phosphate and $1.58 \pm 0.05 \mathrm{~g}$ of sodium hydroxide (both from Sinopharm Chemical Reagent Co., Ltd, China) in $1000 \mathrm{~mL}$ of deionized, degassed water (3). The system was configured with a bath paddle speed of $100 \mathrm{rpm}(1,2)$ at a temperature of $37 \pm 0.5^{\circ} \mathrm{C}$. A 10-mL sample was collected at each time point, diluted 1 in 5, and mixed automatically. Two individually prepared standards (15 mg in $200 \mathrm{~mL}$ ) were diluted manually 1 in 5 . The diluted standards and samples were injected by the system into the UV spectrophotometer, measured at 296 $\mathrm{nm}$, and dissolution results were calculated.

A $1 \%$ standard check was configured to verify the standard measurement. For comparison, samples were taken manually from the bath vessels at exactly the same time they were collected by the system. These samples were diluted and measured manually and compared to results produced by the automated system.

Readings obtained manually by measuring in a $0.3-\mathrm{cm}$ flow cell (Shimadzu Corporation, Kyoto Japan) and from the automated system after preparation (diluted 1 in 5 and mixed) are shown in Table 1. 
Table 1. Results for Two Individually Prepared Standards Measured Manually and with the Automated System after Dilution

\begin{tabular}{|c|c|c|c|c|c|}
\hline \multirow{2}{*}{$\begin{array}{c}\text { Standard } \\
\text { Samples }\end{array}$} & \multicolumn{2}{|c|}{ Manual Sampling } & \multicolumn{2}{|c|}{$\begin{array}{c}\text { Automated Dissolution } \\
\text { System }\end{array}$} \\
\cline { 3 - 5 } & & $\begin{array}{c}\text { Absorbance } \\
\text { (0.3-cm cell) }\end{array}$ & Response & $\begin{array}{c}\text { Absorbance } \\
\text { (diluted } \\
\text { 5-fold, 1-cm } \\
\text { cell) }\end{array}$ & $\begin{array}{c}\text { Response } \\
\text { Difference } \\
\text { (\%) }\end{array}$ \\
\hline Control 1 & 15.05 & 0.594 & \multirow{2}{*}{0.99} & 0.393 & 0.99 \\
\hline Control 2 & 15.06 & 0.599 & & 0.397 & \multirow{2}{*}{} \\
\hline
\end{tabular}

Table 2. Manual and Automated Results for Salicylic Acid Dissolution Analysis

\begin{tabular}{|c|c|c|c|c|c|}
\hline \multirow[b]{2}{*}{$\begin{array}{l}\text { Vessel } \\
\text { No. }\end{array}$} & \multicolumn{2}{|c|}{ Manual Sampling } & \multicolumn{2}{|c|}{$\begin{array}{c}\text { Automated Dissolution } \\
\text { System }\end{array}$} & \multirow[b]{2}{*}{ \% Deviation } \\
\hline & $\begin{array}{l}\text { Absorbance } \\
\text { (0.3-cm cell) }\end{array}$ & $\begin{array}{c}\% \\
\text { Dissolution }\end{array}$ & $\begin{array}{l}\text { Absorbance } \\
\text { (diluted } \\
5 \text {-fold, } \\
1-\mathrm{cm} \text { cell ) }\end{array}$ & $\begin{array}{c}\% \\
\text { Dissolution }\end{array}$ & \\
\hline 1 & 0.729 & 28 & 0.475 & 27 & 1.6 \\
\hline 2 & 0.778 & 30 & 0.505 & 29 & 2.0 \\
\hline 3 & 0.759 & 29 & 0.495 & 28 & 1.5 \\
\hline 4 & 0.724 & 27 & 0.471 & 27 & 1.8 \\
\hline 5 & 0.722 & 27 & 0.466 & 27 & 2.5 \\
\hline 6 & 0.692 & 26 & 0.451 & 26 & 1.6 \\
\hline
\end{tabular}

Table 3. Results for Commercially Available Metformin Hydrochloride 500-mg Extended-Release Tablets from Three Different Manufacturers

\begin{tabular}{|c|c|c|c|c|c|c|}
\hline \multicolumn{7}{|c|}{ Manafacturer } \\
\hline $\begin{array}{c}\text { Sampling } \\
\text { Time }\end{array}$ & Manual & $\begin{array}{c}\text { ASP2000 } \\
\text { System }\end{array}$ & Manual & $\begin{array}{c}\text { ASP2000 } \\
\text { System }\end{array}$ & Manual & $\begin{array}{c}\text { ASP2000 } \\
\text { System }\end{array}$ \\
\hline $15 \mathrm{~min}$ & - & $14 \%$ & - & $12 \%$ & - & $13 \%$ \\
\hline $30 \mathrm{~min}$ & - & $21 \%$ & - & $20 \%$ & - & $23 \%$ \\
\hline $1 \mathrm{~h}$ & $31 \%$ & $31 \%$ & $30 \%$ & $30 \%$ & $33 \%$ & $34 \%$ \\
\hline $2 \mathrm{~h}$ & - & $46 \%$ & - & $44 \%$ & - & $48 \%$ \\
\hline $3 \mathrm{~h}$ & $57 \%$ & $56 \%$ & $54 \%$ & $54 \%$ & $58 \%$ & $59 \%$ \\
\hline $4 \mathrm{~h}$ & - & $65 \%$ & - & $62 \%$ & - & $67 \%$ \\
\hline $6 \mathrm{~h}$ & - & $78 \%$ & - & $75 \%$ & - & $79 \%$ \\
\hline $8 \mathrm{~h}$ & - & $87 \%$ & - & $84 \%$ & - & $87 \%$ \\
\hline $10 \mathrm{~h}$ & $93 \%$ & $93 \%$ & $90 \%$ & $91 \%$ & $91 \%$ & $90 \%$ \\
\hline $12 \mathrm{~h}$ & - & $97 \%$ & - & $94 \%$ & & $92 \%$ \\
\hline $14 \mathrm{~h}$ & - & $100 \%$ & - & $97 \%$ & & $92 \%$ \\
\hline
\end{tabular}

\section{CONCLUSION}

The results of the validation show very good agreement with manual procedures performed using the same stock standard solution. Acceptable repeatability was obtained from multiple measurements. Repeated tests involved loading created standard methods and following the onscreen instructions, which makes the system very robust for use in a QC environment. Reports were professionally presented in different graphical formats, as shown by a typical report graph in Figure 5. In practice, trained analysts should achieve the same results thus eliminating user variability.

Repeatability of results shows low CV, even with dilution ratios of 1 in 100 . The results for all samples at 1,3 , and $10 \mathrm{~h}$ agree within $1 \%$ of the manual method. With the automated system, it was possible to measure more timepoint readings due to a high degree of automation and no analyst intervention, hence the number of sampling times was increased (Table 3). The objective to develop an automated method that could collect and dilute samples and measure automatically was demonstrated and validated.

\section{ACKNOWLEDGMENTS}

The authors gratefully acknowledge the support from Significant New Drugs Creation Special Science and Technology Major (No. 2015ZX09303001).

\section{REFERENCES}

1. Dissolution Test. In Pharmacopeia of the People's Republic of China, Volume II, Appendix X; Pharmacopoeia Commission of the People's Republic of China: Beijing, 2010; pp A105-A107.

2. 2.9.3 Dissolution Test for Solid Dosage Form. In European Pharmacopoeia, 8th ed.; European Directorate for the Quality of Medicines \& Healthcare, Council of Europe: Strasbourg, France, 2012; pp 228-230.

3. $<711>$ Dissolution. In The United States Pharmacopeia and National Formulary USP 36-NF 31; The United States Pharmacopeial Convention, Inc.: Rockville, MD, 2012.

4. Setter, S. M.; Iltz, J. L.; Thams, J.; Campbell, R. K. Metformin hydrochloride in the treatment of type 2 diabetes mellitus: A clinical review with a focus on dual therapy. Clin. Ther. 2003, 25 (12), 2991-3026. DOI: 10.1016/S0149-2918(03)90089-0.

5. Campbell, I. W.; Howlett, H. C. S. Worldwide Experience of Metformin as an Effective Glucose-lowering Agent: A Meta-analysis. Diabetes/Metab. Rev. 1995, 11 (S1), S57-S62. DOI: 10.1002/dmr.5610110509.

6. Metformin Hydrochloride Extended-Release Tablets. In The United States Pharmacopeia and National Formulary USP 33-NF 28; The United States Pharmacopeial Convention, Inc.: Rockville, MD, 2010.

7. Feng, S.; Wang, L.; Wang, J. Determination of metformin hydrochloride enteric-coated tablets by UV. Chin. J. Mod. Appl. Pharm. 2001, 18 (6), 463-465. 